\title{
Clinicopathological characteristics and prognostic risk factors of breast cancer patients with bone metastasis
}

\author{
Yuanxing Pan, Yunfei Lin, Chuan Mi \\ Department of Orthopedics, Peking University First Hospital, Beijing, China \\ Contributions: (I) Conception and design: Y Pan; (II) Administrative support: Y Pan; (III) Provision of study materials or patients: Y Pan; (IV) \\ Collection and assembly of data: Y Lin, C Mi; (V) Data analysis and interpretation: Y Lin, C Mi; (VI) Manuscript writing: All authors; (VII) Final \\ approval of manuscript: All authors. \\ Correspondence to: Yuanxing Pan. Department of Orthopedics, Peking University First Hospital, Beijing 100083, China. Email: 180147569@qq.com.
}

\begin{abstract}
Background: A retrospective study was performed to summarize the clinicopathological characteristics of breast cancer patients with bone metastasis, to clarify the metastasis sites, and to explore the risk factors affecting prognosis.

Methods: Breast cancer patients with bone metastasis diagnosed in our hospital from January 2008 to January 2019 were included. Through follow-up by telephone call or return visit, the metastasis sites and clinicopathological characteristics were summarized. The risk factors influencing prognosis were analyzed by univariate and multivariate regression analyses.

Results: Multifocal bone metastases were dominant in the 150 patients, and the metastatic rates in the spine, chest, pelvis, limbs, and skull were $75.3 \%, 74.0 \%, 56.0 \%, 46.7 \%$, and $28.7 \%$, respectively, with significant differences $(\mathrm{P}<0.01)$. Kaplan-Meier univariate analysis showed that age, menstrual status, number of metastatic lymph nodes, clinical stage, endocrine therapy, alkaline phosphatase level, visceral metastasis, and number of bone metastasis sites affected the overall survival. Cox multivariate regression analysis revealed that endocrine therapy, number of metastatic lymph nodes, visceral metastasis, number of bone metastasis sites, and c-erbB-2 expression were independent prognostic factors.

Conclusions: Middle-aged and elderly patients with breast cancer, mainly aged 40-60 years old, are prone to bone metastasis. The incidence rate of bone metastasis is high within 3 years after surgery, involving the spine, chest, pelvis, limbs, and skull in descending order. The number of metastatic lymph nodes, endocrine therapy, visceral metastasis, number of bone metastasis lesions at the first onset, and c-erbB-2 expression are independent prognostic factors influencing the survival rate of breast cancer patients with bone metastasis.
\end{abstract}

Keywords: Breast cancer; bone metastasis; clinicopathological characteristics; prognosis

Submitted Jun 29, 2021. Accepted for publication Aug 23, 2021.

doi: $10.21037 /$ atm-21-4052

View this article at: https://dx.doi.org/10.21037/atm-21-4052

\section{Introduction}

Breast cancer, as a malignant tumor seriously harming women's health, is known as the number one killer of females. With the development of medical treatments, the quality of life and survival of breast cancer patients have been improved. However, the morbidity and mortality rates of breast cancer remain high. Though the morbidity rate of breast cancer in China is not as high as that in western countries, it has shown a younger trend in urban areas on a larger scale, causing great harm to patients as well as society. Bone metastasis of breast cancer is mainly found in axial bones, of which spinal metastasis is most common $(1,2)$. The bone metastasis of breast cancer is related to the age at the onset. With aging, the incidence rate of bone metastasis keeps rising (3).

With the continuous improvement of early diagnosis methods and comprehensive treatments for breast cancer, the disease-free survival and overall survival have significantly 
increased. Nevertheless, distant metastasis is still a major cause for the high mortality rate of breast cancer (4). About $20-85 \%$ of breast cancer patients suffer from distant metastasis within 5 years after initial diagnosis (5), and around $6-10 \%$ of cases are diagnosed with distant metastasis at the first visit (6). Bone metastasis is the second most common type of advanced breast cancer metastasis following lung metastasis (7). Sundquist et al. reported that approximately $70 \%$ of advanced breast cancer patients suffered from bone metastases in the spine, sternum, ribs, pelvis, limbs, and skull (8). The autopsy report showed that up to $90 \%$ of deaths had bone metastasis (9). Bone pain and osteoporosis are typical first symptoms of bone metastasis. Severe bone pain is often the main reason and purpose of treatment for bone metastasis patients. Some cases suffer from pathological fractures, spinal cord compression, or hypercalcemia, seriously affecting quality of life, as well as being mainly responsible for deaths. However, the currently available studies regarding the bone metastasis of breast cancer did not include sufficient factors (e.g. influence of treatment regimens on the prognosis), and most of them did not consider the impact of ethnicity.

Hence, in this study, the clinical characteristics of breast cancer patients with bone metastasis were summarized, and the factors affecting prognosis were analyzed, aiming to provide evidence for the early prediction of prognosis and prompt treatment. As a result, the life span of patients can be extended, and their quality of life can be improved.

We present the following article in accordance with the STROBE reporting checklist (available at https://dx.doi. org/10.21037/atm-21-4052).

\section{Methods}

\section{Baseline clinical data}

Breast cancer patients with bone metastasis diagnosed in our hospital from January 2008 to January 2019 were included. According to the diagnostic criteria of breast cancer bone metastasis (10), those who met 1 or more of the following three criteria were diagnosed: (I) a definite breast cancer history; (II) clinically suspected bone metastasis with obvious symptoms such as local bone pain, cachexia, and high serum alkaline phosphatase (ALP) level; (III) multiple lesions revealed by bone imaging; (IV) bone metastasis detected by X-ray and/or MRI examination. Patients included in the study met all of the following three criteria: (I) pathologically diagnosed with breast cancer. For those undergoing surgery in other hospitals, a clear pathological diagnosis was confirmed; (II) bone metastasis detected for the first time after breast cancer was diagnosed; (III) complete clinical and pathological data. The exclusion criteria were as follows: (I) breast cancer patients with other malignant tumors; (II) those with incomplete clinicopathological data. The study was conducted in accordance with the Declaration of Helsinki (as revised in 2013). The patients or their families agreed with the study and signed the informed consent, and this study was approved by the Ethics Committee of our hospital (approval No. PUFH200801013).

\section{Distribution of bone metastasis sites}

All included patients were definitely diagnosed with bone metastasis by a radionuclide whole-body scan, X-ray, CT, MRI, or other examinations. Referring to Wilson's method (11), human bones were divided into 5 regions (15 parts in total) to analyze the metastasis sites, namely chest: ribs, collarbone, sternum, and scapula; spine: cervical vertebra, thoracic vertebra, and lumbar vertebra; pelvis: sacrum, ischium, pubis, sacrum, and sacroiliac joint; limbs: upper and lower limbs; skull.

\section{Detection methods}

The expression levels of carbohydrate antigen 15-3 (CA15-3) and carcinoembryonic antigen (CEA) of the 150 patients were measured using the ACS:180SE automated chemiluminescence analyzer. The normal range of serum CA15-3 is $0-28.0 \mathrm{U} / \mathrm{mL}$ and the normal range of CEA is $0-5.2 \mathrm{ng} / \mathrm{mL}$. Therefore, a serum CA15-3 level of $\geq 28 \mathrm{U} / \mathrm{mL}$ and a CEA level of $\geq 5.2 \mathrm{ng} / \mathrm{mL}$ indicated a positive result. Serum ALP level was determined by the 4-nitrophenol phosphate rate method using an automatic biochemical analyzer. The normal range is $38-110 \mathrm{U} / \mathrm{L}$. Therefore, a serum ALP level of $\geq 110$ U/L suggested a positive result. The expression levels of estrogen receptor (ER), progesterone receptor (PR), and c-erbB-2 were detected with the immunohistochemical SP method and evaluated based on immunohistochemical results: the number of $\mathrm{ER}^{+}$and $\mathrm{PR}^{+}$cells of $>10 \%(+)$ indicated a positive result, while that of $<10 \%(+)$ or $(-)$ indicated a negative result. C-erbB-2 (-) and weakly positive $(+)$ indicated a negative result, and $(+++)$ indicated a positive result, but further c-erbB-2 detection was needed by carrying out fluorescence or chromogenic in situ 
hybridization in cases of $(++)$ and (++ to +++$)$. According to the TNM staging system in the sixth edition of the AJCC Cancer Staging Manual (2002), breast cancer is divided into 3 stages, namely primary tumor size of $\leq 2 \mathrm{~cm}$ : T1; 2.1-5 cm: T2; $>5$ cm: T3. No lymph node metastasis pathologically diagnosed after surgery: N0; $1-3$ metastatic lymph nodes: $\mathrm{N} 1 ; \geq 4$ metastatic lymph nodes: $\mathrm{N} 2$ or N3.

\section{Follow-up}

All included patients were followed up by telephone call or return visit, and the survival status was recorded. Survival without bone metastasis referred to the duration from breast cancer surgery to the occurrence of bone metastasis. Overall survival referred to the duration from diagnosis of bone metastasis to death. The surviving patients were followed up until January 2020, and the time of last followup was recorded (months). Patients who died of causes other than breast cancer were recorded as loss to followup (time of last follow-up). Lost and surviving cases were statistically analyzed as censored data.

\section{Establishment of the database}

The sites and characteristics of breast cancer bone metastasis were analyzed. For the survival analysis, the relationships between various clinicopathological parameters and prognosis were evaluated.

\section{Statistical analysis}

All data were statistically analyzed by SPSS 17.0 software. The quantitative data were expressed as mean \pm standard deviation, and intergroup comparisons were performed by the nonparametric test. The Kaplan-Meier method was used for univariate survival analysis, and the survival rates of different groups were compared with the log-rank method. Multivariate survival analysis was conducted by the Cox proportional hazards regression model. $\mathrm{P}<0.05$ was considered statistically significant.

\section{Results}

\section{Baseline clinical data}

The 150 patients were aged $17-70$ years old, with a median age of 47.0 years old, among which $7.3 \%$ (11/150) were aged $17-30$ years old, $23.3 \%$ (35/150) were aged $31-40$ years old,
$33.3 \%$ (50/150) were aged $41-50$ years old, $28.7 \%$ (43/150) were aged 51-60 years old, and $7.3 \%(11 / 150)$ were aged above 60 years old. The incidence of bone metastasis reached the peak at the age of 40-60 years old. Among the 150 patients, Han people accounted for $84.67 \%$ (127/150), Hui people for $12.0 \%$ (18/150), Mongolian people for $1.33 \%$ (2/150), and Manchu people for $2.0 \%$ (3/150). There were 64 premenopausal cases $(42.67 \%)$ and 86 postmenopausal cases $(57.33 \%)$.

Among the 150 patients, the primary breast cancer occurred in the left breast in 81 cases (54.0\%) and in the right breast in 69 cases $(46.0 \%)$. The mass was in the upper outer quadrant of the breast in 70 cases $(46.7 \%)$, the lower outer quadrant in 25 cases $(16.7 \%)$, the upper inner quadrant in 18 cases $(12.0 \%)$, the lower inner quadrant in 8 cases $(5.3 \%)$, and at other sites (around the areola, multiple quadrants) in 29 cases (19.3\%). Among all patients, invasive ductal carcinoma accounted for $76.0 \%$ (114/150), invasive lobular carcinoma for $7.3 \%$ (11/150), early non-invasive or invasive cancer for $12.7 \%$ (19/150), and other types for $4.0 \%(6 / 150)$.

Surgical treatment was performed for 135 out of the 150 patients. The tumor size was $\leq 2 \mathrm{~cm}$ (T1) in 39 cases, $2.1-5 \mathrm{~cm}$ (T2) in 79 cases, and $>5 \mathrm{~cm}$ (T3) in 17 cases. After surgery, it was pathologically confirmed that there was no lymph node metastasis (N0) in 43 cases, 1-3 metastatic lymph nodes (N1) in 32 cases, and $\geq 4$ metastatic lymph nodes (N2 and N3) in 60 cases. The number of metastatic lymph nodes and tumor size were unknown in 15 cases who did not undergo surgery. There were 71 cases in stage I-II, 64 cases in stage III, and 15 cases in stage IV. Moreover, there were 50 ER-negative cases and 100 ER-positive cases, 48 PRnegative cases and $102 \mathrm{PR}$-positive cases, and 70 c-erbB-2negative cases and $46 \mathrm{c}$-erbB-2-positive cases (34 cases were eliminated).

\section{Treatment methods}

Surgical treatment was performed for 135 out of the 150 patients, including 90 cases of modified radical surgery, 30 cases of radical surgery, 1 case of breast-conserving surgery, and 14 cases of tumor resection. The remaining 15 cases did not undergo surgery. A total of 107 patients underwent adjuvant chemotherapy, while 28 patients did not. The chemotherapy regimen was mainly CAF (cyclophosphamide + adriamycin + fluorouracil) or CMF (cyclophosphamide + methotrexate + fluorouracil) and AT (adriamycin + paclitaxel). Among the 135 patients, 52 cases received radiotherapy, while the remaining 83 cases 


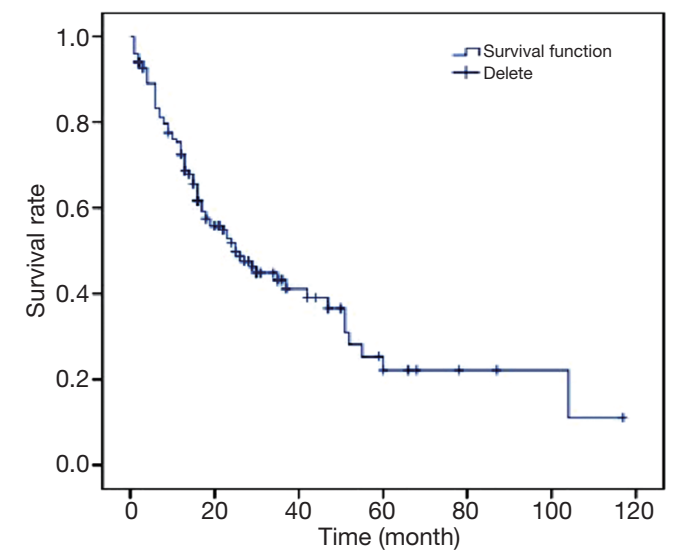

Figure 1 Survival curve of breast cancer patients with bone metastasis.

did not. Endocrine therapy was performed in 59 out of the 150 patients, but was not adopted in the remaining 91 cases.

\section{Bone metastasis-related data}

Among the 150 patients with bone metastasis, $43.33 \%$ $(65 / 150)$ were complicated with visceral metastasis, $56.67 \%$ $(85 / 150)$ had no visceral metastasis, $30.67 \%$ (46/150) had single bone metastasis, $69.33 \%(104 / 150)$ had multiple bone metastases, $46.67 \%$ (70/150) were complicated with ostealgia, and 53.33\% (80/150) had no ostealgia. Among the 135 patients undergoing surgery, bone metastasis occurred in $60.74 \%(82 / 135)$ of cases within 3 years after surgery, and in $39.26 \%(53 / 135)$ of cases after 3 years.

Among the 150 breast cancer patients with bone metastasis, the metastasis occurred at a single site in 46 cases and at multiple sites in 104 cases. Spinal metastasis was the most common [75.3\% (94/150)], followed by chest metastasis [74.0\% (93/150)]. The rates of skull metastasis, limb metastasis, and pelvic metastasis were $28.7 \%$, $46.7 \%$, and $56.0 \%$, respectively. The metastasis rate had statistically significant differences at different sites $\left(\chi^{2}=92.917, \mathrm{P}=0.000\right)$.

\section{Survival of breast cancer patients with bone metastasis}

The median survival time of the 150 breast cancer patients with bone metastasis was $25 \pm 4.512$ months. They were followed up until January 1, 2020, and there were 80 deaths and 70 censored cases. Among the 70 cases, 54 cases were still alive and 16 cases were lost to follow-up. The rate of loss to follow-up was $10.6 \%(16 / 150)$. The 1-, 3-, and 5-year survival rates were $72.4 \%, 43.2 \%$, and $22.2 \%$, respectively. The cumulative survival rate and survival curve of the 150 breast cancer patients with bone metastasis are shown in Figure 1.

\section{Univariate analysis of factors affecting prognosis}

The Kaplan-Meier method was used for the survival analysis of 150 breast cancer patients with bone metastasis. Covariates included clinical parameters (age at the time of diagnosis with breast cancer, postoperative stage, pathological type, number of metastatic axillary lymph nodes, tumor size, hormone receptor status, visceral metastasis status, number of lesions at the onset of bone metastasis, and duration from bone metastasis to surgery), the levels of CA15-3, CEA, and ALP in peripheral blood at the time of bone metastasis, and treatment-related parameters (endocrine therapy for bone metastases).

\section{Relationship of general information with prognosis}

The results of the log-rank test revealed that there was no statistically significant difference in the survival rate of breast cancer patients with bone metastasis among different ethnic groups $(\mathrm{P}>0.05)$. The survival rate of patients at different ages and menstrual status had statistically significant differences $(\mathrm{P}<0.05)$ (Table 1 and Figure 2).

\section{Relationship of clinicopathological characteristics with prognosis}

The results of the log-rank test showed that the survival rate of breast cancer patients with bone metastasis had no statistically significant differences under different pathological types and tumor sizes $(\mathrm{P}>0.05)$, but had statistically significant differences under different numbers of metastatic lymph nodes and stages $(\mathrm{P}<0.05)$ (Table 2 and Figure 3).

\section{Relationship of hormone receptors with prognosis}

According to the results of the log-rank test, the survival rate of breast cancer patients with bone metastasis had no statistically significant differences between ER- and PRnegative and positive groups $(\mathrm{P}>0.05)$, but had a statistically significant difference between c-erbB-2-negative and positive groups $(\mathrm{P}<0.05)$ (Table 3 and Figure 4$)$.

\section{Relationship between characteristics of bone metastasis in breast cancer and prognosis}

Among the 150 breast cancer patients with bone metastasis, 
Table 1 Relationship of general information with prognosis

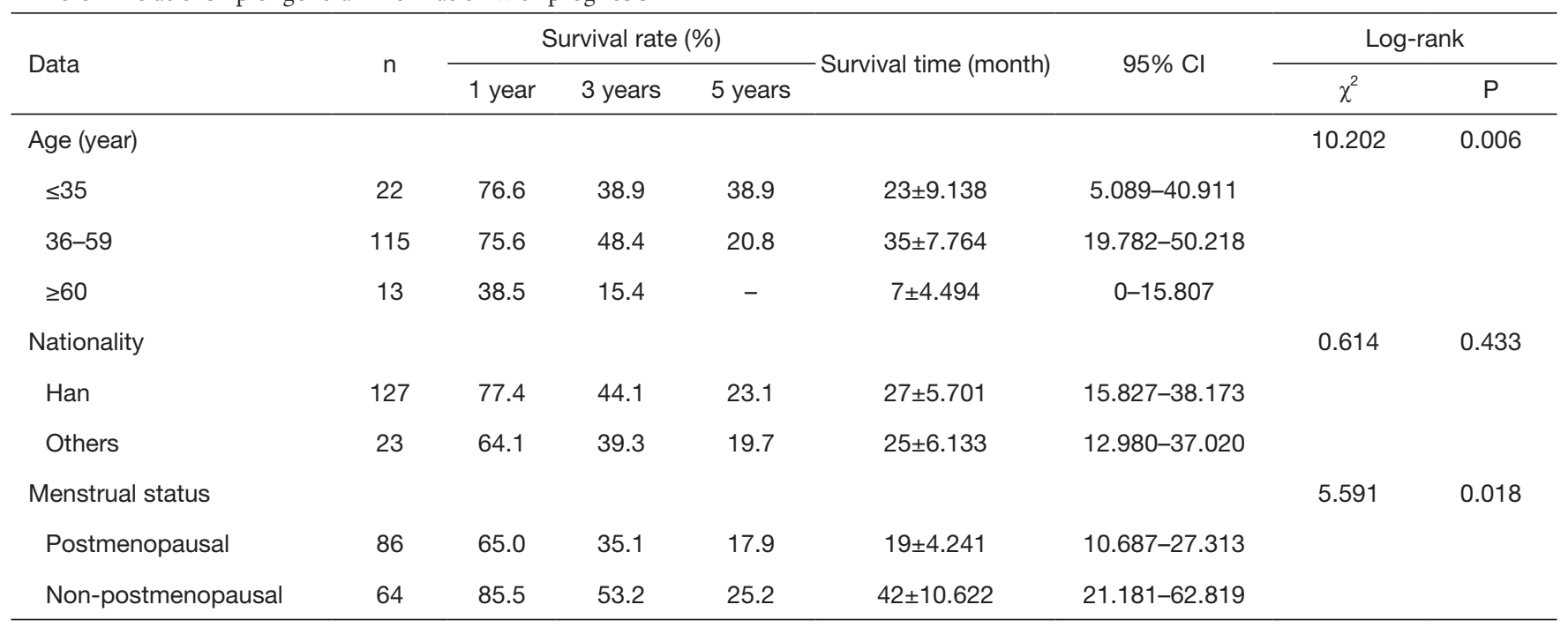
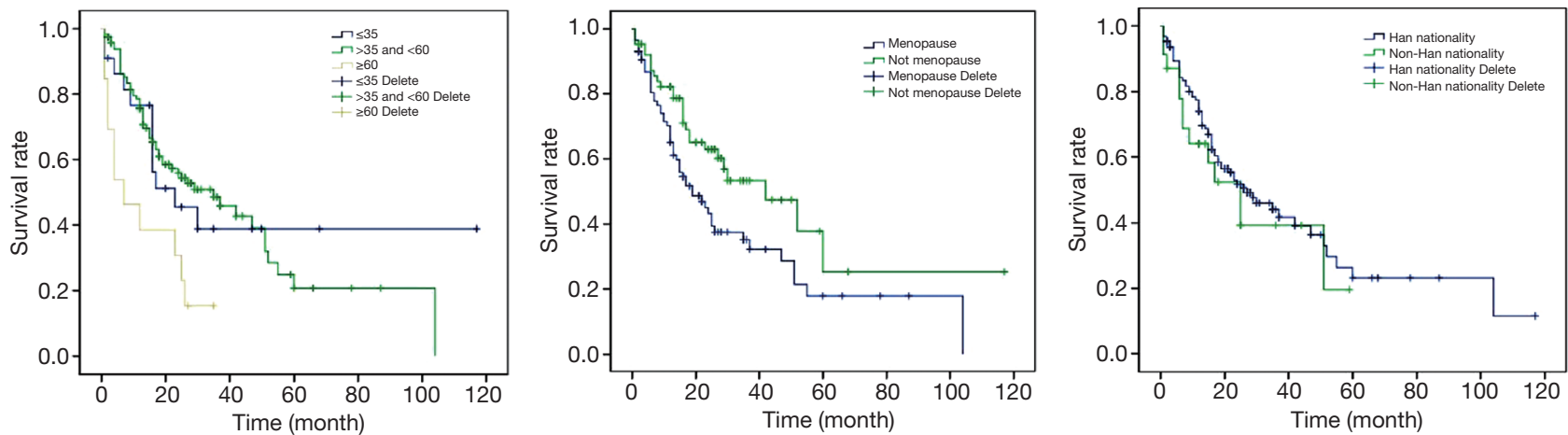

Figure 2 Survival curves of patients with different ages, nationalities, and menstrual statuses.

70 cases experienced ostealgia, which did not occur in the remaining 80 cases. The results of the log-rank test showed that the survival rate had no statistically significant difference between the two groups $(\mathrm{P}>0.05)$. A total of 85 cases had bone metastasis alone, and the remaining 65 cases were complicated with visceral metastasis. The results of the log-rank test showed that the survival rate had a statistically significant difference between the two groups $(\mathrm{P}<0.05)$. Surgical treatment was performed for 135 out of the 150 patients, among which bone metastasis occurred in 53 cases within 3 years after surgery, and 82 cases after 3 years. The survival rate had no statistically significant difference between the two groups $(\mathrm{P}>0.05)$. Furthermore, metastasis occurred at a single site in 46 cases and at multiple sites in 104 cases, and the 2 groups had a statistically significant difference in the survival rate $(\mathrm{P}<0.05)$ (Table 4 and Figure 5).

\section{Relationship of treatment methods with prognosis}

Endocrine therapy was performed in 59 patients, while the remaining 91 patients did not undergo this treatment. The survival rate had a statistically significant difference between the two groups ( $\mathrm{P}=0.025)$ (Table 5 and Figure 6).

Relationship between peripheral blood CA15-3, CEA, and ALP levels during bone metastasis and prognosis

The results of the log-rank test showed that among the 150 patients with bone metastasis, the level of CA15-3 was $\leq 100 \mathrm{U} / \mathrm{mL}$ in 111 cases and $>100 \mathrm{U} / \mathrm{mL}$ in 39 cases, and the two groups had a statistically significant difference in the survival rate $(\mathrm{P}<0.05)$. The level of CEA was $\leq 5.2 \mathrm{ng} / \mathrm{mL}$ in 94 cases and $>5.2 \mathrm{ng} / \mathrm{mL}$ in 56 cases, and the two groups had no statistically significant difference in the survival rate $(\mathrm{P}>0.05)$. The level of ALP was $\leq 110 \mathrm{U} / \mathrm{L}$ in 87 cases and 
Page 6 of 14

Pan et al. Bone metastasis of breast cancer

Table 2 Survival rate and clinicopathological characteristics of breast cancer patients with bone metastasis

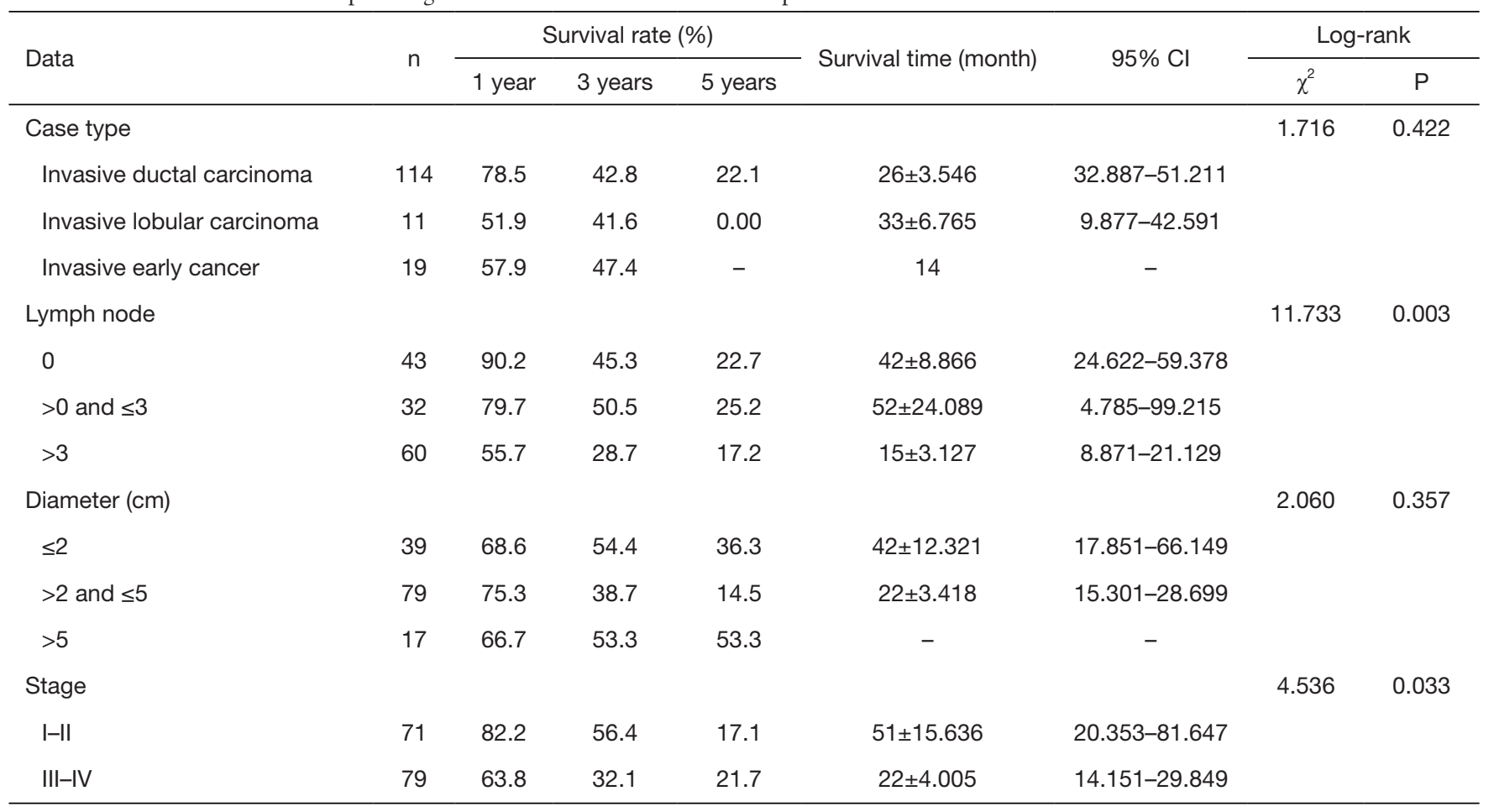
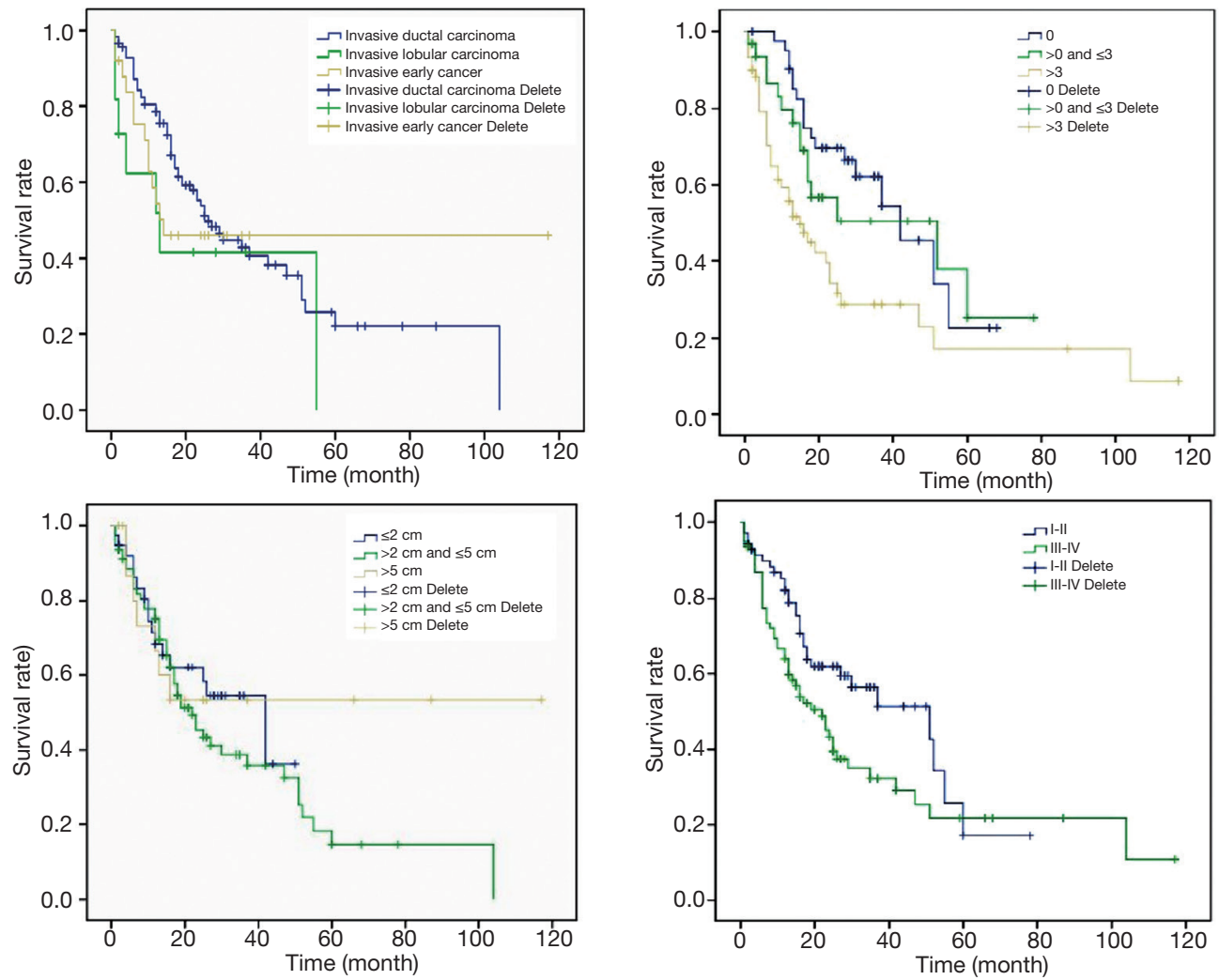

Figure 3 Survival curves of patients with different pathological types, numbers of metastatic lymph nodes, tumor sizes, and stages. 
Table 3 Relationship of hormone receptors with prognosis

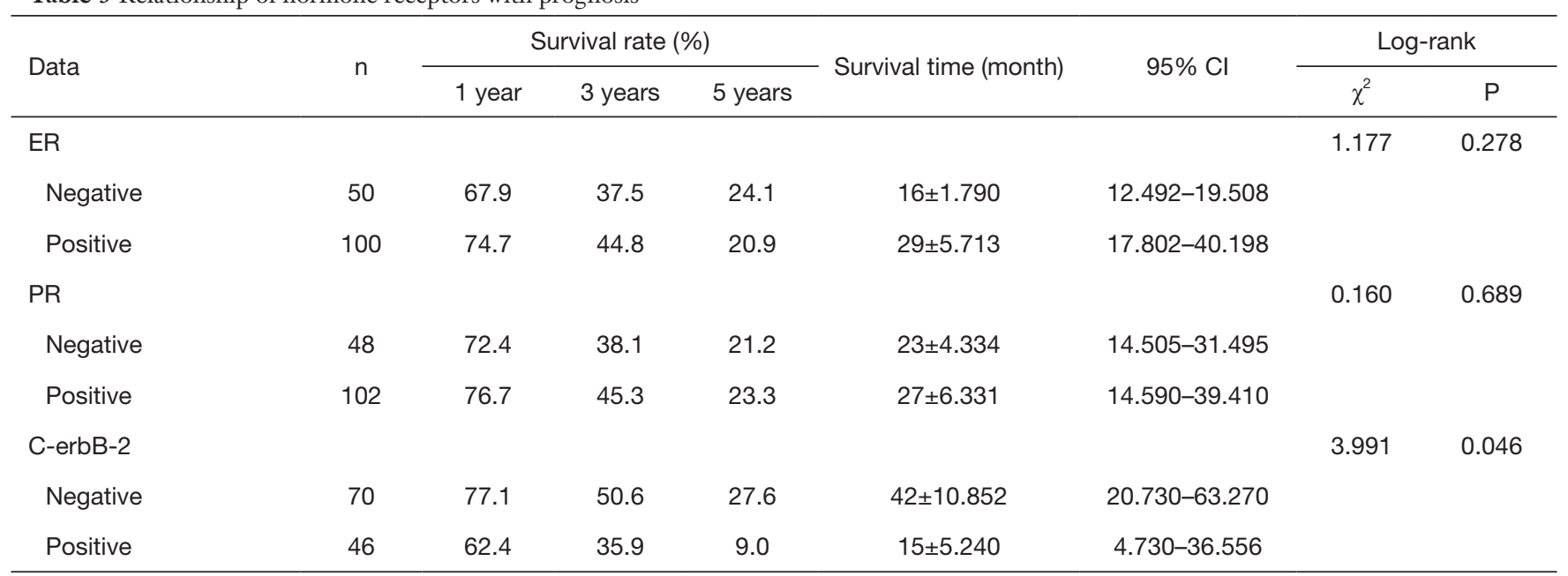

$\mathrm{ER}$, estrogen receptor; PR, progesterone receptor.
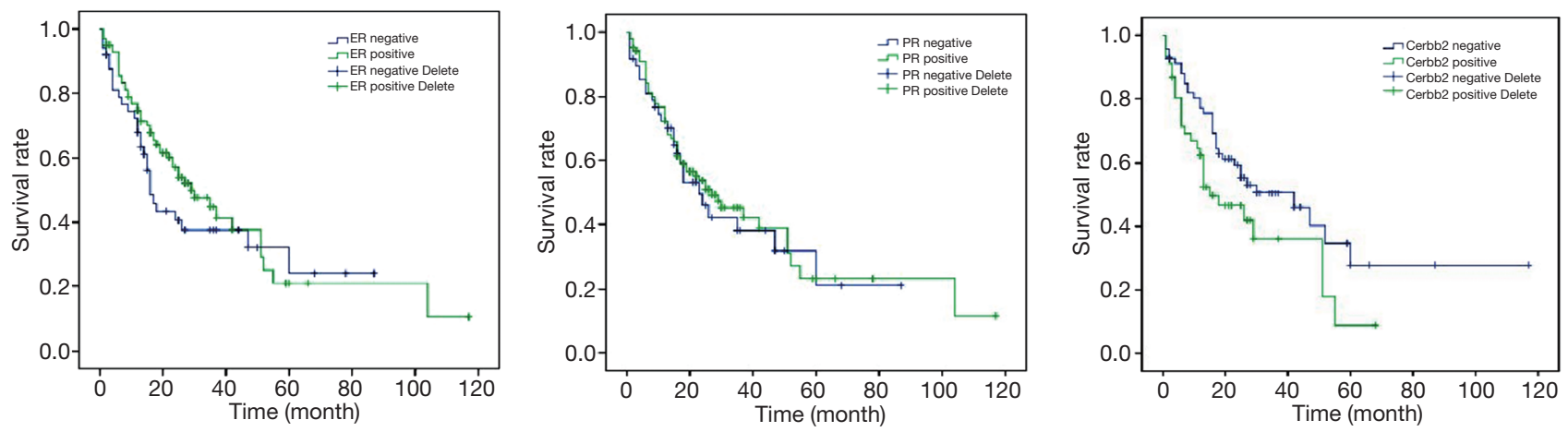

Figure 4 Relationship of hormone receptors with prognosis.

$>110 \mathrm{U} / \mathrm{L}$ in 63 cases, and the survival rate had a statistically significant difference between the two groups $(\mathrm{P}<0.05)$ (Table 6 and Figure 7).

\section{Cox regression analysis of factors affecting prognosis}

According to the Cox regression analysis, the number of bone metastases, the number of metastatic lymph nodes, whether endocrine therapy was performed, whether visceral metastasis was complicated, and c-erbB-2 status were the influencing factors for the prognosis of breast cancer patients with bone metastasis (Table 7).

\section{Discussion}

As one of the most common malignant tumors in women, breast cancer has shown an increasing incidence rate in many countries in recent years. With the advances in medical treatments, the quality of life and survival time of patients have been improved to a large extent, but the morbidity and mortality rates of breast cancer remain high. Metastasis and recurrence are still the main culprits for the failed treatment of breast cancer. Distant metastasis is a major cause for death in breast cancer patients, mostly in the bone, lung, liver and brain. The recurrence and metastasis location of breast cancer are closely related to the prognosis. Visceral metastasis, especially liver metastasis, is an index for the poor prognosis of metastatic breast cancer (12). Geiger et al. reported that the median survival time of visceral involvement group at the first metastasis was significantly shorter than that of non-visceral involvement group, and visceral metastasis was an independent poor prognostic 
Page 8 of 14

Pan et al. Bone metastasis of breast cancer

Table 4 Relationship between characteristics of bone metastasis in breast cancer and prognosis

\begin{tabular}{|c|c|c|c|c|c|c|c|c|}
\hline Data & $\mathrm{n}$ & \multicolumn{3}{|c|}{ Survival rate (\%) } & $\begin{array}{l}\text { Survival time } \\
\text { (month) }\end{array}$ & $95 \% \mathrm{Cl}$ & \multicolumn{2}{|c|}{ Log-rank } \\
\hline Ostealgia & & & & & & & 0.194 & 0.660 \\
\hline Yes & 70 & 74.4 & 41.0 & 21.0 & $25 \pm 5.795$ & $13.642-36.358$ & & \\
\hline No & 80 & 70.8 & 46.0 & 23.0 & $27 \pm 8.471$ & $10.396-43.604$ & & \\
\hline Yes & 65 & 66.8 & 35.0 & 13.2 & $18 \pm 3.944$ & $10.269-25.731$ & & \\
\hline No & 85 & 76.6 & 49.8 & 34.2 & $30 \pm 13.020$ & $4.481-55.519$ & & \\
\hline $\begin{array}{l}\text { Time from surgery to bone } \\
\text { metastasis (years) }\end{array}$ & & & & & & & 3.606 & 0.058 \\
\hline Single & 46 & 86.1 & 66.3 & 16.4 & $51 \pm 6.735$ & $37.799-64.201$ & & \\
\hline Multiple & 104 & 66.6 & 31.4 & 20.9 & $19 \pm 2.588$ & 13.928-24.072 & & \\
\hline
\end{tabular}
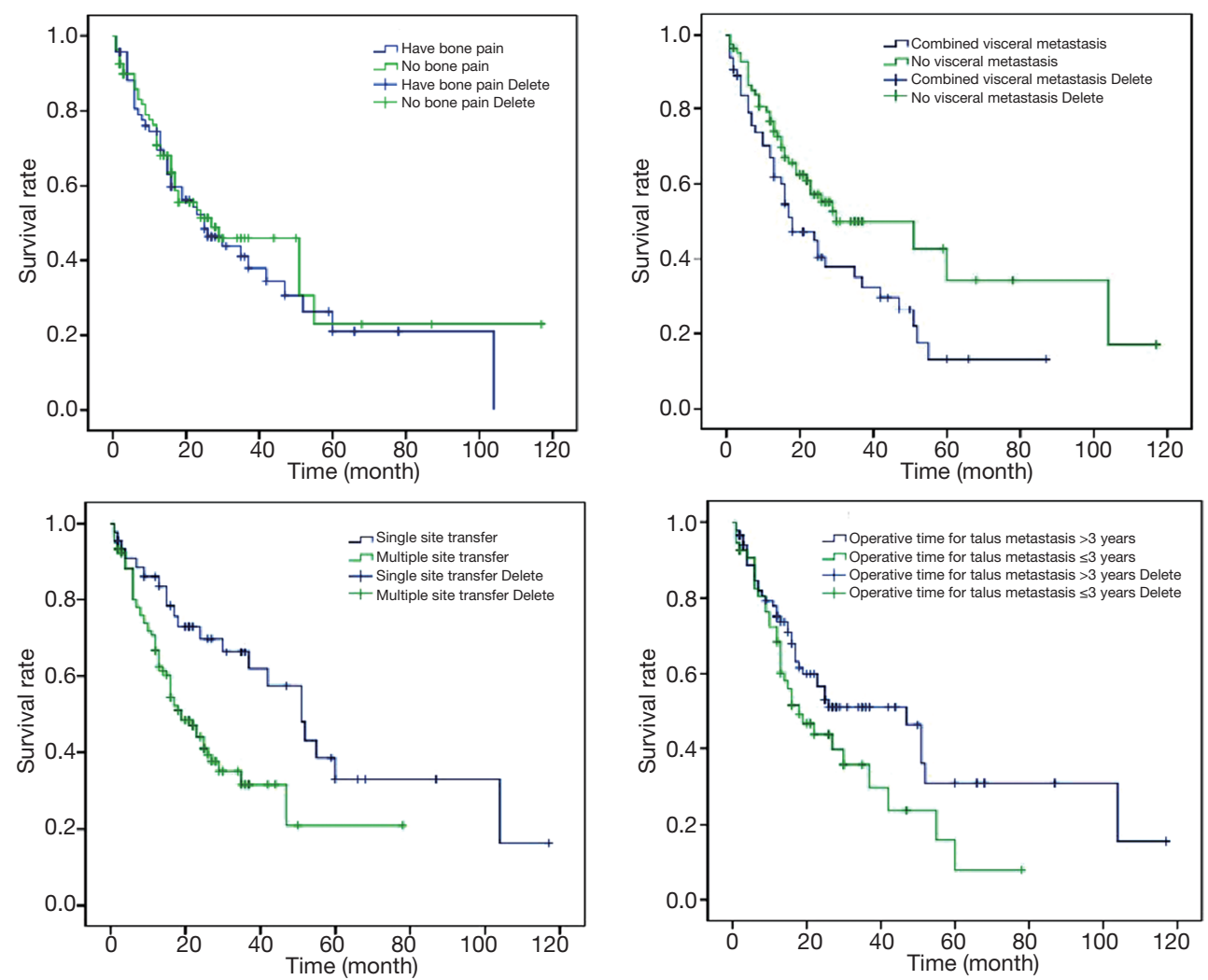

Figure 5 Relationship between characteristics of bone metastasis in breast cancer and prognosis. 
Table 5 Relationship of treatment methods with prognosis

\begin{tabular}{|c|c|c|c|c|c|c|c|c|}
\hline Endocrine therapy & $\mathrm{n}$ & \multicolumn{3}{|c|}{ Survival rate (\%) } & Survival time (month) & $95 \% \mathrm{Cl}$ & \multicolumn{2}{|c|}{ Log-rank } \\
\hline Yes & 59 & 65.6 & 57.7 & 28.6 & $42 \pm 13.447$ & $15.644-68.356$ & 5.040 & 0.025 \\
\hline No & 91 & 68.7 & 33.9 & 17.8 & $23 \pm 3.394$ & $16.347-29.653$ & & \\
\hline
\end{tabular}

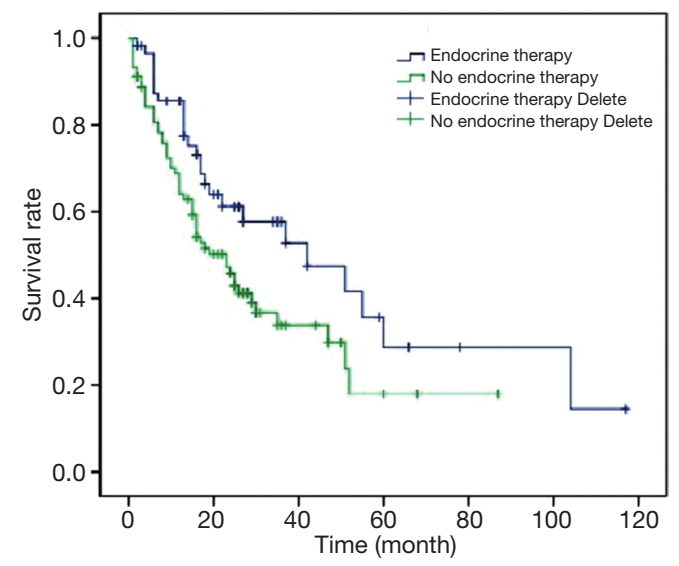

Figure 6 Relationship of treatment methods with prognosis.

factor (13). Coleman et al. found that the median survival time of patients with multiple or visceral metastasis was $>22$ months, while such time of patients without visceral metastasis was $>33$ months. Among them, patients with bone metastases had the best prognosis (14). Liu et al. retrospectively analyzed 135 patients with metastatic breast cancer, and found that the 1-, 2- and 5-year survival rates were $53.3 \%, 25.2 \%$, and $1.5 \%$, respectively. Among them, ER status, disease-free survival, number of metastatic sites and liver metastasis were important factors affecting the prognosis (15). Besides, Er et al. retrospectively analyzed 2193 patients with metastatic breast cancer from 1973 to 2003. Among them, 132 cases of metastatic breast cancer were first found in the liver, with the range being significantly related to the prognosis (16).

Bone tissues are one of the most common sites for distant metastasis of breast cancer. The occurrence of skeletal-related events (SREs) causes great pain to patients and even threatens life, seriously affecting the quality of life and survival time of breast cancer patients. Therefore, early detection, early diagnosis, and early treatment of bone metastases are extremely important, and individualized treatment of patients with bone metastasis is crucial. Accordingly, the clinical characteristics of bone metastasis in breast cancer were summarized in this study, the patients' survival statuses were followed up, and the factors affecting the prognosis of breast cancer patients with bone metastasis were analyzed, so as to guide clinicians to judge, predict, and evaluate the prognosis of patients and take active therapeutic measures as soon as possible.

The distribution of bone metastases in breast cancer patients has certain characteristics and patterns. The results of most studies (17) have shown that bone metastasis in breast cancer is dominated by the axial skeleton, with spinal metastasis being the most common, followed by chest metastasis, while skull and limb metastases are relatively rare. In this study, the results showed that 94 out of the 150 breast cancer patients $(75.3 \%)$ with bone metastasis suffered from spinal metastasis, followed by chest metastasis $(74.0 \%)$. The rates of pelvic metastasis, limb metastasis, and skull metastasis were $56.0 \%, 46.7 \%$, and $28.7 \%$, respectively. The metastasis rate had statistically significant differences at different sites $(\mathrm{P}<0.01)$. The reasons for such patterns for the sites of bone metastasis in breast cancer may include the following: (I) the sites of bone metastasis in breast cancer are related to Batson's plexus (18). The spinal venous system has a wide range of branches and complex connections to the portal vein, vena cava, azygos vein, and pulmonary vein. Moreover, the spinal venous system is characterized by no venous valve and low venous pressure. Breast cancer cells invade the intercostal veins first and then flow directly into the superior vena cava via the azygos vein or accessory azygos vein, and they can also flow into the spinal venous system. In the case of elevated abdominal pressure, the blood between the spinal venous system and vena cava can flow into each other, so the spine is the most common site of metastasis. In addition, blood can reflow into the pelvis, causing pelvic metastasis. (II) Different metastasis sites are related to the blood supply of bones. Bones with abundant blood supply create a breeding ground for cancer cells, so they are more prone to metastasis.

Both single lesion or multiple lesions can exist at the onset of bone metastasis in breast cancer, but multiple lesions are more dominant. Koizumi et al. found that among 
Table 6 Relationship between peripheral blood CA15-3, CEA, and ALP levels during bone metastasis and prognosis

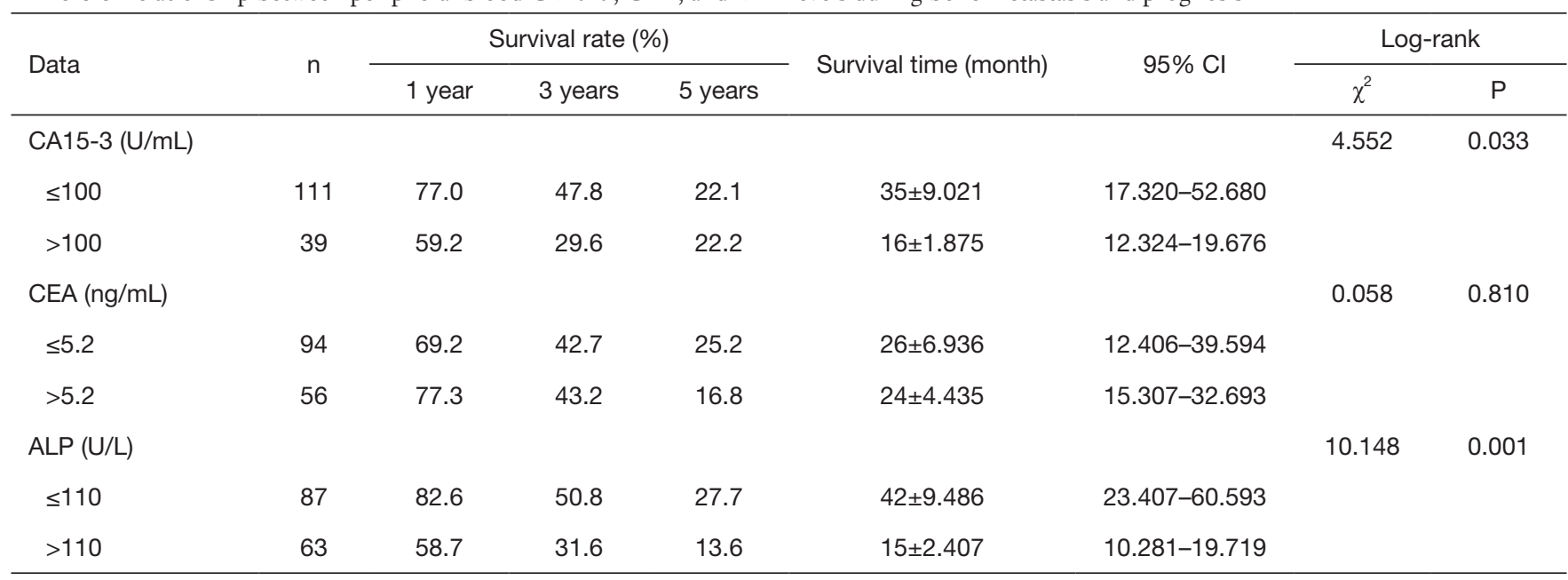
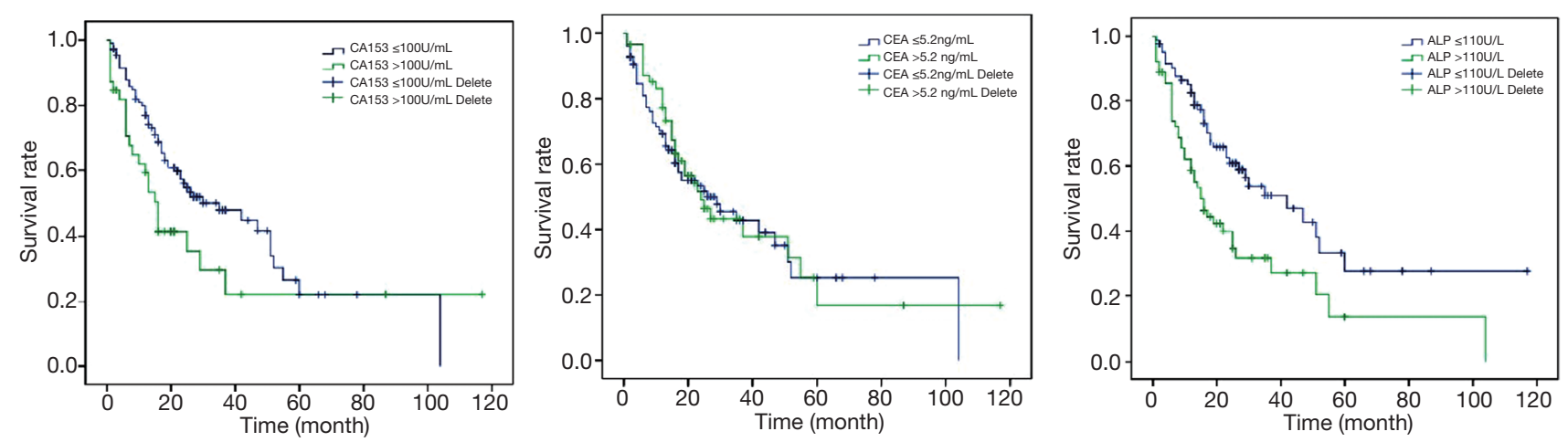

Figure 7 Relationship between peripheral blood CA15-3, CEA, and ALP levels during bone metastasis and prognosis. ALP, Alkaline phosphatase; CA15-3, carbohydrate antigen 15-3; CEA, carcinoembryonic antigen.

Table 7 Cox regression analysis of factors affecting prognosis

\begin{tabular}{|c|c|c|c|c|c|c|c|}
\hline \multirow{2}{*}{ Prognosis factors } & \multirow{2}{*}{$\beta$} & \multirow{2}{*}{ SE } & \multirow{2}{*}{ Wald } & \multirow{2}{*}{$\mathrm{P}$} & \multirow{2}{*}{$\operatorname{Exp}(\beta)$} & \multicolumn{2}{|c|}{$95 \% \mathrm{Cl}$} \\
\hline & & & & & & Lower & Upper \\
\hline Number of metastasis sites & 0.782 & 0.377 & 4.311 & 0.038 & 2.187 & 1.045 & 4.577 \\
\hline Number of metastatic lymph nodes & 0.343 & 0.168 & 4.136 & 0.042 & 1.409 & 1.013 & 1.960 \\
\hline C-erbB-2 status & 0.813 & 0.286 & 8.112 & 0.004 & 2.256 & 1.289 & 3.948 \\
\hline Complication with visceral metastasis & -0.780 & 0.296 & 6.965 & 0.008 & 0.458 & 0.257 & 0.818 \\
\hline
\end{tabular}

703 breast cancer patients with bone metastasis, 414 cases (59.0\%) had multiple lesions at the onset (19). Boxer et al. reported that the proportion of multiple lesions was higher, with up to $79.0 \%$ of 150 breast cancer patients with bone metastasis having multiple lesions at the onset, and they had a worse prognosis than those with a single lesion (20). In this study, multiple lesions were dominant among the 150 breast cancer patients with bone metastasis (46 cases of 
a single lesion and 104 cases of multiple lesions), similar to the above research results. There was a statistically significant difference in the survival rate between patients with a single lesion at the onset and those with multiple lesions at the onset $(\mathrm{P}<0.05)$. The results of Cox multivariate analysis confirmed that the number of bone metastases at the onset was an independent influencing factor for the prognosis of breast cancer patients with bone metastasis, and patients with a single lesion had a better prognosis than those with multiple lesions.

The clinical manifestations of breast cancer patients with bone metastasis vary based on the bone metastasis site and the degree of bone damage. There are often no specific clinical manifestations at the early stage. Disease progression can manifest as ostealgia, pathological fractures, spinal cord compression, spinal nerve compression signs, and hypercalcemia. Ostealgia is one of the most common clinical manifestations of breast cancer patients with bone metastasis, which can be intermittent or continuous, and often becomes worse at night, seriously affecting the quality of life of patients. There are few reports regarding the association between ostealgia and the prognosis of breast cancer patients with bone metastasis. In this study, it was found that ostealgia had no significant effect on the prognosis of breast cancer patients with bone metastasis.

There are many influencing factors for the prognosis of breast cancer patients with bone metastasis, among which visceral metastasis is generally considered as an important factor affecting prognosis. It is believed by most researchers that the prognosis of breast cancer patients with bone metastasis and visceral metastasis is worse than that of patients with bone metastasis alone, and patients with bone metastasis alone have a longer course of disease and a slower progression of disease (21). In this study, among the 150 patients, 85 cases had bone metastasis alone, and the remaining 65 cases were complicated with visceral metastasis or other tissue metastases, including multiple visceral metastases in some cases. There was a statistically significant difference in the survival rate between patients with bone metastasis alone and those with bone metastasis and visceral metastasis or other tissue metastases $(\mathrm{P}<0.05)$. The results of Cox multivariate analysis showed that visceral metastasis was an independent influencing factor for the prognosis of breast cancer patients with bone metastasis. In other words, the prognosis of breast cancer patients with bone metastasis and visceral metastasis is worse than that of patients with bone metastasis alone.

Bone metastasis in breast cancer is a systemic disease.
The main goals of treatment are to prevent and treat SRE, relieve pain, improve quality of life, and prolong survival time, and systemic treatment-based individualized comprehensive therapy is adopted. Systemic chemotherapy, endocrine therapy, and molecular targeted therapy are the basic drug therapies for bone metastasis in breast cancer. Bisphosphonates are used to prevent and treat SRE, and surgery and radiotherapy are also performed to better control the local symptoms of bone metastasis. The relationship between treatment methods and the prognosis of breast cancer patients with bone metastasis currently remains controversial. In this study, 59 patients with a median survival time of $42 \pm 13.447$ months received endocrine therapy, while the remaining 91 patients with a median survival time of $23 \pm 3.394$ months did not. The survival rate had a statistically significant difference between the two groups $(\mathrm{P}=0.025)$. Furthermore, the results of the Cox multivariate analysis revealed that the relative risk was 1.682 , and the difference was statistically significant between patients undergoing endocrine therapy and those not undergoing endocrine therapy $(\mathrm{P}=0.040)$. Thus, endocrine therapy is an independent influencing factor for the prognosis of breast cancer patients with bone metastasis, and the prognosis of patients receiving endocrine therapy is better.

CA15-3 is a type of glycoprotein antigen closely related to breast cancer. It is released by cancer cells into the blood, and is used for breast cancer monitoring, screening, and the evaluation of prognosis (22). In this study, the survival rate had a statistically significant difference between patients with CA15-3 $\leq 100 \mathrm{U} / \mathrm{mL}$ and those with CA15-3 >100 U/mL $(\mathrm{P}=0.033)$, indicating that $\mathrm{CA} 15-3>100 \mathrm{U} / \mathrm{mL}$ in peripheral blood may be an adverse factor for the prognosis of breast cancer patients with bone metastasis. CEA is an acid glycoprotein with human embryonic antigen-specific determinants, and is a better tumor marker for efficacy determination, disease development and monitoring, and prognosis evaluation of colorectal cancer, breast cancer, and lung cancer. However, it has lower specificity and sensitivity $(23,24)$. The correlation between the level of CEA in peripheral blood and the prognosis of bone metastasis in breast cancer has rarely been reported. In this study, the results showed that among all 150 patients, the positive rate of CEA was $37.33 \%(56 / 150)$, and its lowest, highest, and median values were $0.4,>839.2$, and $3.31 \mathrm{ng} / \mathrm{mL}$, respectively, which were all at normal levels (the normal value of serum CEA determined by radioimmunoassay is $5.2 \mathrm{ng} / \mathrm{mL}$ ). There was no statistically significant difference in the survival rate 
between patients with CEA $\leq 5.2 \mathrm{ng} / \mathrm{mL}$ and those with CEA $>5.2 \mathrm{ng} / \mathrm{mL}(\mathrm{P}>0.05)$, suggesting that the level of CEA in peripheral blood cannot serve as an independent index for predicting the prognosis of breast cancer patients with bone metastasis.

ALP is widely present in various organs of the human body, and is most abundant in the liver followed by the kidneys, and is also common in bones, intestines, and placenta. Schindler et al. compared 62 patients with malignant tumors and 40 adults without a history of tumors, and concluded that the sensitivity and specificity of ALP in the diagnosis of bone metastasis were $52 \%$ and $100 \%$, respectively, and the ALP level could be used as a good index for determining the presence or absence of disease progression in cancer patients with bone metastasis (25). In this study, the level of ALP was $\leq 110 \mathrm{U} / \mathrm{L}$ in 87 cases and $>110 \mathrm{U} / \mathrm{L}$ in 63 cases, and the survival rate had a statistically significant difference between the two groups $(\mathrm{P}<0.05)$. It was confirmed by Cox regression analysis that serum ALP was not an independent influencing factor for the prognosis of breast cancer patients with bone metastasis.

At present, the bone metastasis of breast cancer is clinically treated by systemic therapy (chemotherapy, endocrine therapy or drug therapy) and local therapy (radiotherapy) (26). Concurrent radiotherapy and chemotherapy are commonly used to treat advanced breast cancer with bone metastasis, which can relieve the pain and reduce the incidence of pathological fractures. Nevertheless, this method induces a series of adverse reactions while killing cancer cells (27). Bisphosphonates can be selectively absorbed by osteoclasts, which inhibit their activity, induce the apoptosis and then suppress bone resorption. Zoledronic acid, which is currently the most pharmacologically active bisphosphonate, can effectively inhibit the precursor cells of osteoclasts and thus reduce the damage to bone trabeculae (28). Hence, it is now well-accepted that zoledronic acid combined with concurrent radiotherapy and chemotherapy have satisfactory therapeutic effects on the bone metastasis of advanced breast cancer.

The routine prognosis and recurrence predictors of breast cancer include ER, PR and HER2. Based on this and the molecular classification of breast cancer, endocrine therapy or targeted HER2 therapy is selected for patients. However, the above methods have disadvantages such as number of sampling, detection methods and strong subjectivity of diagnosis. Although micrometastatic cells in bone marrow, i.e. diffuse tumor cells, have independent prognostic value for breast cancer patients, more invasive bone marrow aspiration is required. In contrast, the detection of circulating tumor markers (including CTCs, ctDNA, ctDNA methylation, exosomes and TEP) is less traumatic, and many types of markers can be measured at the same time, providing more comprehensive disease information (29).

In conclusion, bone metastasis in breast cancer frequently occurs in middle-aged and elderly people, mainly at the age of 40-60 years old. The incidence rate of bone metastasis is high within 3 years after surgery, and the metastasis sites are mainly the spine, chest, pelvis, limbs, and skull. The number of metastatic lymph nodes, endocrine therapy, the presence of visceral metastasis, the number of bone metastases at the onset, and the expression of c-erbB-2 are independent influencing factors for the survival rate of breast cancer patients with bone metastasis. Chen et al. also analyzed the clinicopathological factors related to the bone metastasis of breast cancer, but did not comprehensively analyze their effects on the prognosis of patients. In the meantime, they did not include factors such as ethnicity and treatment regimen (30). Since this is a respective study, there may be deviations in our results. Further multicenter studies with larger sample sizes are ongoing in our group to verify the conclusion.

\section{Acknowledgments}

Funding: None.

\section{Footnote}

Reporting Checklist: The authors have completed the STROBE reporting checklist. Available at https://dx.doi. org/10.21037/atm-21-4052

Data Sharing Statement: Available at https://dx.doi. org/10.21037/atm-21-4052

Conflicts of Interest: All authors have completed the ICMJE uniform disclosure form (available at https://dx.doi. org/10.21037/atm-21-4052). The authors have no conflicts of interest to declare.

Ethical Statement: The authors are accountable for all aspects of the work in ensuring that questions related to the accuracy or integrity of any part of the work are appropriately investigated and resolved. The study was conducted in accordance with the Declaration of Helsinki (as revised in 2013). The patients or their families agreed with 
the study and signed the informed consent, and this study was approved by the Ethics Committee of our hospital (approval No. PUFH200801013).

Open Access Statement: This is an Open Access article distributed in accordance with the Creative Commons Attribution-NonCommercial-NoDerivs 4.0 International License (CC BY-NC-ND 4.0), which permits the noncommercial replication and distribution of the article with the strict proviso that no changes or edits are made and the original work is properly cited (including links to both the formal publication through the relevant DOI and the license). See: https://creativecommons.org/licenses/by-nc-nd/4.0/.

\section{References}

1. Ooi LL, Zheng Y, Stalgis-Bilinski K, et al. The bone remodeling environment is a factor in breast cancer bone metastasis. Bone 2011;48:66-70.

2. Stathopoulos GP, Trafalis D, Kaparelou M. Bone metastasis in breast cancer is treated by high-dose tamoxifen. J BUON 2016;21:1013-5.

3. Søe K, Plesner T, Jakobsen EH, et al. Is retention of zoledronic acid onto bone different in multiple myeloma and breast cancer patients with bone metastasis? J Bone Miner Res 2013;28:1738-50.

4. Svetlovska D, Mardiak J. Treatment strategy of early-stage breast cancer. Bratisl Lek Listy 2005;106:362-5.

5. Greenberg PA, Hortobagyi GN, Smith TL, et al. Longterm follow-up of patients with complete remission following combination chemotherapy for metastatic breast cancer. J Clin Oncol 1996;14:2197-205.

6. Eivazy P, Atyabi F, Jadidi-Niaragh F, et al. The impact of the codelivery of drug-siRNA by trimethyl chitosan nanoparticles on the efficacy of chemotherapy for metastatic breast cancer cell line (MDA-MB-231). Artif Cells Nanomed Biotechnol 2017;45:889-96.

7. Pimentel I, Lohmann AE, Ennis M, et al. A phase II randomized clinical trial of the effect of metformin versus placebo on progression-free survival in women with metastatic breast cancer receiving standard chemotherapy. Breast 2019;48:17-23.

8. Sundquist M, Brudin L, Tejler G. Improved survival in metastatic breast cancer 1985-2016. Breast 2017;31:46-50.

9. Martin TJ, Moseley JM. Mechanisms in the skeletal complications of breast cancer. Endocr Relat Cancer 2000;7:271-84.

10. Zuo CT, Yin DC, Fan HX, et al. Study on diagnostic value of P1NP and $\beta$-CTX in bone metastasis of patients with breast cancer and the correlation between them. Eur Rev Med Pharmacol Sci 2019;23:5277-84.

11. Wilson MA, Calhoun FW. The distribution of skeletal metastases in breast and pulmonary cancer: concise communication. J Nucl Med 1981;22:594-7.

12. Insa A, Lluch A, Prosper F, et al. Prognostic factors predicting survival from first recurrence in patients with metastatic breast cancer: analysis of 439 patients. Breast Cancer Res Treat 1999;56:67-78.

13. Geiger S, Cnossen JA, Horster S, et al. Long-term followup of patients with metastatic breast cancer: results of a retrospective, single-center analysis from 2000 to 2005. Anticancer Drugs 2011;22:933-9.

14. Coleman RE, Smith P, Rubens RD. Clinical course and prognostic factors following bone recurrence from breast cancer. Br J Cancer 1998;77:336-40.

15. Liu MT, Huang WT, Wang AY, et al. Prediction of outcome of patients with metastatic breast cancer: evaluation with prognostic factors and Nottingham prognostic index. Support Care Cancer 2010;18:1553-64.

16. Er O, Frye DK, Kau SW, et al. Clinical course of breast cancer patients with metastases limited to the liver treated with chemotherapy. Cancer J 2008;14:62-8.

17. Solomayer EF, Diel IJ, Meyberg GC, et al. Metastatic breast cancer: clinical course, prognosis and therapy related to the first site of metastasis. Breast Cancer Res Treat 2000;59:271-8.

18. Onken JS, Fekonja LS, Wehowsky R, et al. Metastatic dissemination patterns of different primary tumors to the spine and other bones. Clin Exp Metastasis 2019;36:493-8.

19. Koizumi M, Yoshimoto M, Kasumi F, et al. Comparison between solitary and multiple skeletal metastatic lesions of breast cancer patients. Ann Oncol 2003;14:1234-40.

20. Boxer DI, Todd CE, Coleman R, et al. Bone secondaries in breast cancer: the solitary metastasis. J Nucl Med 1989;30:1318-20.

21. Rades D, Fehlauer F, Schulte R, et al. Prognostic factors for local control and survival after radiotherapy of metastatic spinal cord compression. J Clin Oncol 2006;24:3388-93.

22. Chen WZ, Shen JF, Zhou Y, et al. Clinical characteristics and risk factors for developing bone metastases in patients with breast cancer. Sci Rep 2017;7:11325.

23. Sütterlin M, Oehler MK, Caffier H. Clinical value of CYFRA 8/18 and TPS in the diagnosis and follow up of invasive breast cancer. Anticancer Res 1997;17:2963-5.

24. Moro D, Vuillez JP, Brichon PY, et al. 854 Two-step 
immunoscintigraphy for non-small cell lung cancer staging using a bispecific anti-CEA/anti-indium-DTPA antibody and an indium-111-labeled DTPA dimer. Lung Cancer 1997;18:219-20.

25. Schindler F, Lajolo PP, Pinczowski H, et al. Bone and total alkaline phosphatase for screening skeletal metastasis in patients with solid tumours. Eur J Cancer Care (Engl) 2008;17:152-6.

26. Su YC, Choi WH, Chan HP. Treatment of Bone metastasis in Breast Cancer. Korean J Clin Oncol 2008;4:15-9.

27. Haffty BG. Concurrent chemo-radiotherapy in operable breast cancer. Women Oncol Rev 2011;6:85-7.

28. Szafran AA, Folks K, Warram J, et al. Death receptor 5 agonist TRA8 in combination with the bisphosphonate

Cite this article as: Pan Y, Lin Y, Mi C. Clinicopathological characteristics and prognostic risk factors of breast cancer patients with bone metastasis. Ann Transl Med 2021;9(16):1340. doi: $10.21037 / \mathrm{atm}-21-4052$ zoledronic acid attenuated the growth of breast cancer metastasis. Cancer Biol Ther 2009;8:1109-16.

29. Sollier-Christen E, Liu HE, Vuppalapaty M, et al. EGFR mutational detection in vortex-enriched CTCs, ctDNA, and comparison to tumor tissue in non-small-celllung-cancer (NSCLC) patients. Am Assoc Cancer Res 2018;78:1598.

30. Chen J, Zhu S, Xie XZ, et al. Analysis of clinicopathological factors associated with bone metastasis in breast cancer. J Huazhong Univ Sci Technolog Med Sci 2013;33:122-5.

(English Language Editor: C. Betlzar) 